\title{
Eficácia de programas de educação para adultos portadores de Hipertensão Arterial
}

\author{
Efficiency of health education programs for adults with high blood pressure
}

Eficacia de programas de educación en salud para adultos con Alta Presión

\section{Emília Soares Chaves}

Enfermeira. Doutoranda. Professora Substituta do Departamento de Enfermagem da Universidade Federal do Ceará-UFC, Fortaleza, CE. Bolsista CAPES. emilly.e@zipmail.com.br

Ingrid Martins Leite Lúcio

Enfermeira. Doutoranda. Professora substituta do Departamento de Enfermagem da UFC,

Fortaleza, CE. Bolsista CAPES ingrid lucio@yahoo.com.br

Thelma Leite de Araújo

Enfermeira. Doutora. Docente do Departamento de Enfermagem da UFC, Fortaleza, CE.

Pesquisadora do CNPq. thelma@ufc.br

Marta Maria Coelho Damasceno

Enfermeira. Doutora. Docente do Departamento de Enfermagem da UFC, Fortaleza, CE.

Pesquisadora do CNPq. martadamasceno@terra.com.br

\footnotetext{
Submissão : 06/09/2005
}

Aprovação: 18/03/2006

\section{RESUMO}

É importante conhecer como estratégias de educação em saúde, utilizadas por enfermeiros, para portadores de hipertensão arterial vêem se desenvolvendo, e se são realmente eficazes para esses indivíduos. Objetivou-se analisar estudos que enfocassem estratégias para desenvolver educação em saúde com adultos portadores de hipertensão arterial. Pesquisa bibliográfica, utilizando a base de dados MEDLINE. Obteve-se uma relação de resumos, utilizando os descritores: hipertensão, educação e enfermagem. Ao final, dois artigos na língua inglesa atenderam aos critérios estabelecidos. Pôde-se verificar que programas estruturados levam a melhorias nas condições de saúde, tanto no que se refere aos fatores de risco para hipertensão como para a adesão ao tratamento instituído, havendo considerável mudança de comportamento e melhoria da adesão ao tratamento medicamentoso.

Descritores: Pressão alta; Enfermagem; Educação em saúde.

\section{ABSTRACT}

It is of great importance to acknowledge health education strategies nurses use on patients with highblood pressure, seeing how they respond and if the method is eficient for for them. The aim was to analise researches which focused on strategies to develop health education for adults with highblood pressure. This is a bibliography study which used data basis from MEDLINE. The abstracts found showed the folowing themes: highblood pressure, education and nursing. After the analisis two articles in english fitted in the stablished criteria. It was noticed that organized programes improve health conditions in factors such as highblood pressure risks and aproval to the treatment given, being noticed a change of behavior and adherence to the drug treatment.

Descriptors: High pressure; Nursing; Health education.

\section{RESUMEN}

Es importante conocer como estrategias de educación en salud, utilizadas por enfermeros, para portadores de hipertensión arterial ven desarrollándose, y se son realmente eficaces para esos individuos. Se objetivó analizar estudios que enfocaran estrategias para desarrollar educación en salud con adultos portadores de hipertensión arterial. Investigación bibliográfica, utilizando la base de datos MEDLINE. Se obtuvo una relación de resúmenes, utilizando los descriptores: hipertensión, educación y enfermería. Al final, dos artículos en la lengua inglesa atendieron a los criterios establecidos. Se pudo verificar que programas estructurados llevan a mejorías en las condiciones de salud, tanto en lo que se refiere a los factores de riesgo para hipertensión como para la adhesión al tratamiento instituido, habiendo considerable cambio de comportamiento y mejoría de la adhesión al tratamiento medicamentoso.

Descriptores: Alta presión; Enfermería; Educación en salud.

Chaves ES, Lúcio IML, Araújo TL, Damasceno MMC. Eficácia de programas de educação em saúde para portadores de hipertensão arterial. Rev Bras Enferm 2006 jul-ago; 59(4): 543-7.

\section{INTRODUÇÃO}

Há algumas décadas, as doenças do aparelho circulatório ou cardiovasculares são a primeira causa de morte no Brasil(1). Dentro deste contexto, a hipertensão arterial representa uma das situações clínicas que atingem 0 aparelho circulatório, e também, é um dos principais fatores de risco para outras doenças como as cerebrovasculares, as vasculares, as isquêmicas do coração e a diabetes mellitus, contribuindo para elevar os índices de morbidade e mortalidade ${ }^{(2,3)}$.

Sua prevalência atinge $20 \%$ da população adulta mundial e se acredita que o mesmo ocorra no Brasil(4). Apresenta-se como uma síndrome caracterizada pela presença de níveis de pressão arterial elevados, associados a alterações hormonais e, no metabolismo, a fenômenos tróficos ${ }^{(5)}$. Quando se 
encontra em estágio avançado, proporciona lesões graves em órgãosalvo como coração, rins, retina, cérebro, que podem levar o indivíduo à dependência física ou até a morte. Da mesma forma que em outras partes do mundo, faz-se importante o seu reconhecimento como um grande problema de saúde no Brasil.

A hipertensão arterial está associada à presença de diversos fatores de risco, como hereditariedade, sedentarismo, tabagismo, etilismo, ingestão elevada de sal e obesidade. O sucesso no seu tratamento inclui, além da utilização correta do medicamento, a mudança dos hábitos de vida referentes aos fatores citados.

Por ser a hipertensão arterial uma doença multifatorial, 0 desenvolvimento e a implementação de estratégias de intervenção, em particular, aquelas de educação em saúde, envolvem uma ótica ampla, na qual devem ser considerados aspectos individuais e coletivos.

A educação em saúde tem contribuído significativamente para a prevenção e controle de doenças nos últimos 20 anos, principalmente quando se relaciona com os custos para a saúde, os quais podem ser reduzidos por meio dessa estratégia. Sua proposta é fornecer conhecimento com a finalidade de estimular pacientes para efetivar mudanças em seu comportamento(6).

Uma das características da maioria dos processos educativos relacionados à hipertensão arterial, é que, havendo uma equipe multiprofissional atuante, as abordagens diferem, assim como a linguagem. No entanto, observa-se que predomina nos programas a visão reducionista do papel do paciente, desconsiderando, muitas vezes, suas opiniões, crenças e dificuldades ${ }^{(6)}$. Em relação a ações formais de tratamento e acompanhamento para pacientes portadores de hipertensão arterial, têmse conhecimento do Programa de Assistência ao Hipertenso e Diabético do Ministério da Saúde, no qual estão incluídas ações multidisciplinares, dentre elas as que são realizadas pelo enfermeiro, por meio da consulta de enfermagem. Este profissional além de integrar programas desta natureza pode vir a planejar e desenvolver atividades dirigidas ao indivíduo com hipertensão arterial em outros âmbitos, como na rede hospitalar, no atendimento domiciliário, e em universidades, tanto na área do ensino como no campo da pesquisa. Neste último, se destacam os grupos de pesquisa responsáveis por grande parte dessas ações, principalmente preventivas e de educação em saúde.

A enfermeira exerce papel importante dentro do contexto da hipertensão arterial, abrangendo aspectos que vão desde a participação em programas de detecção precoce, até o desenvolvimento de estratégias para garantir adesão ao tratamento. Isto tem levado a um maior esforço no desenvolvimento de estudos, enfocando a educação e orientação do cliente como parte integrante do cuidado de enfermagem ${ }^{(7)}$.

Em geral, são poucos os estudos que avaliam a eficácia das estratégias de educação em saúde utilizadas pelos enfermeiros para portadores de hipertensão arterial. Os estudos abordam de uma forma mais específica um ou outro aspecto importante para o controle da hipertensão arterial, como por exemplo, atividade física ou hábitos alimentares, ou enfocam a hipertensão em conjunto com outras doenças, como diabetes mellitus e problemas cardíacos já instalados. Além disso, abordam muito mais 0 processo de orientação do que a avaliação da eficácia das intervenções realizadas.

Portanto, faz-se importante o desenvolvimento de pesquisas que busquem conhecer como as estratégias de educação em saúde vêem se desenvolvendo, e se são realmente eficazes para os indivíduos com hipertensão arterial( $^{(8)}$.

O presente trabalho surgiu a partir da realização de um Seminário que abordou a Prática Baseada em Evidência na Enfermagem como parte do conteúdo teórico da disciplina "Análise da Pesquisa em Enfermagem", do curso de Doutorado em Enfermagem da Universidade Federal do Ceará. Teve como objetivo analisar estudos que enfocassem estratégias para desenvolver educação em saúde com adultos portadores de hipertensão arterial, visto que o enfermeiro desempenha um papel essencial no seu controle e tratamento identificando fatores que inibem ou acentuam o processo educativo, devido ao fato de ter contato regular com o paciente, posição esta que permite conhecer não apenas suas necessidades educacionais, mas também perceber se esses indivíduos encontram-se motivados para aquisição de novas informações.

Com esse propósito foi estabelecida a seguinte questão: qual a eficácia de programas de educação em saúde para adultos portadores de hipertensão arterial?

\section{METODOLOGIA}

Trata-se de uma pesquisa bibliográfica, já que foi desenvolvida com base em material já elaborado ${ }^{9}$, constituído de artigos científicos. Procurouse analisar material cujo enfoque fosse os resultados obtidos com programas de educação em saúde para adultos portadores de hipertensão arterial.

A procura pelo material bibliográfico para análise foi realizada nas línguas inglesa, portuguesa e/ou espanhola abordando a questão do estudo. Para tanto, foi consultada a base de dados MEDLINE de onde se obteve uma relação de resumos, utilizando os descritores: hipertensão, educação e enfermagem. Essa base é uma das utilizadas freqüentemente por enfermeiros, sendo uma das maiores do mundo. Abrange todas as áreas da literatura biomédica e corresponde ao Index Medicus, com cobertura adicional de literatura de enfermagem e odontologia. Todos os periódicos atualmente indexados no Internacional Nursing Index achamse incluídos no Medline ${ }^{(9)}$.

De início, a procura dos artigos por meio do acesso ao site www.bireme.br obedeceu a seguinte seqüência de links: literatura científica, ciências da saúde, medline (período1993-2004). Na delimitação deste período, procedeu-se a busca utilizou-se apenas o descritor "hipertensão", tendo sido encontradas 634 referências. Como 0 interesse era também em artigos que enfocassem a prática dos enfermeiros, foi associado o descritor "enfermagem", o que reduziu o número de referências para 43. Considerando a necessidade de atender à questão norteadora do estudo, foi incluído um terceiro descritor, "educação", sintetizando 17 referências. Ao obtê-las, a princípio, na forma de resumos, verificou-se que se tratavam de artigos, e, portanto, a partir de então se determinou que estes deveriam atender aos seguintes critérios:

- Envolver descrições da prática de enfermagem relacionadas à educação em saúde com indivíduos adultos, incluindo idosos, portadores de hipertensão arterial sem co-morbidades associadas;

- Permitir identificar a formação dos autores, tendo pelo menos um enfermeiro no rol desses autores;

- Ser estudos clínicos controlados.

Ao analisar as 17 referências (resumos) obtidas, segundo os critérios mencionados, chegou-se aos seguintes resultados:

- 10 não eram estudos clínicos controlados;

- 2 apresentaram a hipertensão arterial associada a outras doenças;

- 3 não tinham o enfermeiro como, pelo menos, um dos autores;

- 2 atenderam aos critérios estabelecidos.

Obteve-se na íntegra os dois artigos que atenderam aos critérios estabelecidos para a análise, sendo ambos na língua inglesa. Após uma leitura criteriosa dos mesmos optou-se por apresentar os dados conforme o modelo de Yeh, Eisenberg, Kaptchuk e Russel(10), que se compõe de: referência, desenho, amostra, intervenção, controle, resultados. Além destes aspectos, também foram regatados os objetivos de cada artigo, local e duração do estudo e tratamento dos dados.

\section{RESULTADOS}

Conforme descrito, foram analisados dois artigos que tratavam de 
intervenções junto a pacientes portadores de hipertensão arterial. Relacionou-se o conteúdo de cada um ao contexto abordado ${ }^{(12)}$. Os dois artigos selecionados para o presente estudo foram obtidos na íntegra sendo ambos na língua inglês, sendo identificados pelos seus autores.

\section{Apresentação dos Dados}

Artigo de Saounatsou et $\mathbf{a l}^{(12)}$.

- Hipótese: Clientes com hipertensão arterial seguiriam melhor seu regime medicamentoso se recebessem instruções sobre adesão dadas por enfermeiros?

- Objetivo: examinar a relação entre a educação de pacientes portadores de hipertensão arterial em acompanhamento de seu regime medicamentoso e variáveis externas (anos de escolaridade, duração do tratamento e seguimento do regime medicamentoso).

- Local e duração do estudo: desenvolvido em dois Hospitais Gerais em Athens e dois Centros de Saúde na cidade de Attica, num período de quatro meses.

- Desenho: Quantitativo; experimental; randomizado.

- Amostra: 40 pacientes adultos diagnosticados com hipertensão arterial. Grupo controle (20) e experimental (20). Critério de inclusão: homem ou mulher; de 30 a 65 anos; diagnóstico de hipertensão idiopática não controlada; uso de 1 ou 2 drogas prescritas; 6 a 12 anos de escolaridade; sem avaliação da adesão no domicílio.

- Intervenção: Programa estruturado centrado nas necessidades do paciente portador de hipertensão arterial.

- Tratamento dos dados: para a análise dos dados foi utilizado o programa estatístico Statistical Package for Social Sciences (SPSS). Para confirmação da hipótese do estudo foi utilizado o teste não paramétrico de Mann-Whitney, e para a investigação da relação das variáveis "anos de escolaridade", "duração da terapia" e "seguimento/adesão" foi utilizado 0 teste de Spearman.

- Resultados: Foi encontrada uma relação estatisticamente significante apenas entre escolaridade e adesão ao tratamento. O programa aplicado por enfermeiros trouxe significativa melhora na adesão dos pacientes em relação ao regime medicamentosos.

O plano do programa educacional aplicado teve como fundamentos teóricos o Modelo do Auto-Cuidado de Orem (1985), o Modelo de Crenças em Saúde de Rosenstock (1974) e o Modelo de Crenças em Saúde de Becker (1974. Como estratégias junto ao grupo experimental, foram realizadas de 4 a 5 visitas domiciliares individuais e contatos por telefone, duas vezes por semana, para enfatizar a importância do acompanhamento, e ao final ainda foi distribuído a cada participante um Kit educativo denominado "Meditos", bem como instruções para seu uso adequado. Esta etapa do estudo foi realizada por quatro enfermeiras, membros do grupo de pesquisa, uma para cada clínica antihipertensiva pertencentes ao estudo Esta medida foi adotada para evitar prejuízos à pesquisa. $\mathrm{A}$ avaliação dos dados foi conduzida por um membro do grupo de pesquisa que não participou das etapas anteriores, ou seja do planejamento e da execução da pesquisa. Quanto às relações entre as variáveis do estudo, apenas foi encontrada relação estatisticamente significante entre escolaridade e adesão ao tratamento.

Os autores deste trabalho confirmaram a hipótese de que clientes hipertensos seguiriam melhor seu regime medicamentoso se recebessem instruções sobre adesão dadas por enfermeiros, através da metodologia proposta. Tendo como enfoque central a variável "adesão ao tratamento medicamentoso" para a investigação da hipótese do estudo, adotaram parâmetros de mensuração estatística com o teste não-paramétrico de Mann-Whitney, no qual o nível de adesão foi classificado em uma escala ordinal de 5 pontos de acordo com o número de pílulas que o paciente esquecia de tomar.

Não foi feita, no estudo, menção quanto a limitações ou dificuldades, mas apontaram sugestões para a obtenção de melhores resultados como a observação dos pacientes em intervalos regulares, número maior da amostra, realização de um pré-teste e uma correlação entre melhoria da adesão e qualidade de vida. Além disso, julgam necessário examinar a influência do programa educacional para diferentes doenças, avaliando também a adesão com o regime medicamentoso.

\section{Artigo de Zernike et $\mathbf{a l}^{(6)}$.}

- Hipótese: a implementação de ações de educação centrada nas necessidades dos pacientes é mais efetiva para a melhoria do conhecimento da hipertensão arterial que informações prestadas de rotina durante a hospitalização.

- Objetivo: examinar a efetividade de duas estratégias, uma que já faz parte do cuidado de um hospital e outra potencialmente eficaz para ser adotada pelos enfermeiros.

- Local e duração do estudo: desenvolvido em três unidades médicas do Royal Brisbome Hospital durante o período de seis meses.

- Desenho: Quantitativo; experimental; randomizado.

- Amostra: 40 pacientes adultos portadores de hipertensão arterial. Grupo controle (20) e experimental (20). Critérios de inclusão: Diagnóstico prévio ou no momento da admissão de hipertensão arterial; prescrição de medicamento antihipertensivo, sendo os próprios pacientes responsáveis por tomar sua medicação.

- Intervenção: Programa dirigido para adesão à terapêutica medicamentosa de pacientes com hipertensão.

- Tratamento dos dados: os escores do pré e pós testes de cada paciente foram comparados usando o teste $t$, verificando se houve diferença estatisticamente significante.

- Resultados: Foi encontrada diferença estatisticamente significante entre pré-teste e pós-teste nos pacientes nos quais foram aplicados o programa educacional estruturado.

Os autores confirmaram que a implementação de ações de educação centrada nas necessidades do pacientes é mais efetiva para a melhoria do conhecimento da hipertensão arterial que informações prestadas de rotina durante a hospitalização. As ações educativas foram planejadas tendo como ponto de partida o levantamento de dados por meio de uma avaliação referente aos hábitos de vida do paciente e, de acordo com as necessidades "problemas" identificadas, as ações foram estabelecidas e efetuadas

A escolha por se trabalhar com mudanças relacionadas ao estilo de vida foi tomada consultando especialistas na área de hipertensão arterial e referencial como The National Heart Foundation. Foram realizados um pré-teste, 24 horas após a admissão do paciente no hospital e dois póstestes, um no momento da alta e outro oito semanas após a alta, por meio de entrevista pelo telefone. Um terceiro contato ainda foi possível com 12 dos 20 integrantes do grupo experimental, que revelaram que 0 nível de conhecimento ainda era significativo mesmo um ano depois

A ação educativa centrada nas necessidades do paciente foi construída a partir da constatação de que materiais educativos entregues como um procedimento de rotina parecem não facilitar 0 aprendizado do paciente fato este, verificado através da revisão de programas existentes. O propósito deste tipo de estratégia foi de minimizar este fato, voltar-se para as reais necessidades do paciente e também ser capaz de identificar se o paciente tem idéias preconcebidas quanto ao direcionamento do seu estado de saúde.

Os autores retrataram o envolvimento dos enfermeiros locados no cenário deste estudo. Os staffs foram treinados sobre seus papéis especíicos na promoção e educação em saúde e como o pesquisador estabeleceria o contato com eles tanto para orientá-los como para tornarse ciente quanto ao momento da alta dos pacientes envolvidos na pesquisa, já que este não se fazia presente de maneira integral no cenário pesquisado. Também conduziram uma avaliação inicial sobre 0 
conhecimento dos pacientes para estimulá-los a questionar sobre o tema hipertensão arterial.

Tendo como enfoque central a variável "nível de conhecimento", relacionado aos fatores de risco para hipertensão arterial, para a investigação da hipótese do estudo, adotaram como parâmetros de mensuração estatística o teste $t$ para comparar os escores do pré e pósteste de cada paciente. 0 nível de conhecimento foi mensurado por escores relativos ao número de respostas corretas. 0 pré-teste e os dois pós-testes foram aplicados a ambos os grupos.

Junto ao grupo teste, foi conduzida uma avaliação oral centrada nas necessidades do paciente relacionadas aos fatores de risco e com base naquelas alteradas, para complementar 0 aprendizado, foi entregue um material em forma de cartões referente aos aspectos que mereciam maior atenção por parte do paciente. 0 grupo controle continuou recebendo as informações que já faziam parte das atividades de rotina da enfermagem.

A estratégia utilizada favoreceu mais a interação enfermeira-paciente na promoção e cuidado com a saúde. Os autores reconheceram como dificuldade do estudo o desenvolvimento da avaliação oral, pois as respostas eram documentadas durante a conversação e revistas várias vezes a fim de tornarem-se mais claras e completas possíveis, e imediatamente após a sessão, o pesquisador necessitava graduar o nível de conhecimento do paciente. Outra limitação do estudo foi a ausência de evidências sobre a fidedignidade das respostas nas mudanças de alguns hábitos, como tabagismo e etilismo.

\section{COMENTÁRIOS}

A educação em saúde é reconhecida pelo seu potencial para a redução de custos junto a diversos contextos da assistência, por favorecer a promoção do auto-cuidado e o desenvolvimento da responsabilidade do paciente sobre decisões relacionadas à saúde. A educação do paciente é vista como questão importante, entretanto a efetividade de estratégias utilizadas para tal fim vem merecendo debates. A enfermagem apresenta forte potencial para a identificação apropriada dessas estratégias ${ }^{(6)}$.

Pelo objetivo do estudo, pôde-se verificar que programas estruturados de educação em saúde para adultos portadores de hipertensão arterial levam a melhorias nas condições de saúde destes indivíduos, tanto no que se refere aos fatores de risco como para a adesão ao tratamento instituído. Foi verificado que nos dois artigos apresentados, houve, após a realização de programas educacionais, considerável mudança de comportamento e melhoria da adesão ao tratamento medicamentoso. Reafirmam as melhorias proporcionadas pela educação em saúde como estratégia junto aos pacientes adultos portadores de hipertensão arterial. No entanto, também é referido que essas ações não são tão fáceis de serem implementadas, por diversos motivos como o método utilizado, a própria interação com os sujeitos, entre outros.

No que diz respeito à realidade brasileira, é sabido que existem ações voltadas para a hipertensão arterial na rede básica de atenção primária. Um exemplo que pode ser citado são as ações inseridas dentro do Programa Saúde da Família (PSF) com a consulta ao paciente com hipertensão e estratégias de educação em saúde para estes indivíduos. O PSF é uma estratégia de assistência à comunidade que consiste em desenvolver ações de promoção, proteção à saúde e assistência numa abordagem multiprofissional, introduzida pelo Ministério da Saúde ${ }^{(13)}$. No entanto, mesmo sem existir estudos controlados que avaliem esse Programa, a experiência de alguns enfermeiros com a clientela tem mostrado que em sua maioria funcionam de forma dependente e se apóiam em um e outro membro da equipe de saúde. Além disso, o motivo dos profissionais que atuam nos PSFs, por exemplo, nem sempre permite concluir que estes mostram-se realmente comprometidos em melhorar as condições de saúde da população assistida. Um estudo revelou que, para médicos, o fator que mais contribuiu para sua inserção no Programa foi a remuneração, e entre os enfermeiros foi por ter sido a primeira possibilidade de emprego após a formatura e ser uma atividade bem remunerada ${ }^{(13)}$. Ademais, a capacitação desses profissionais para este tipo de trabalho, na maioria das vezes, só acontece quando eles já estão atuando junto à comunidade.

Ainda é notório o elevado número de casos de não adesão ao tratamento, principalmente medicamentoso, da hipertensão arterial, incluindo também não seguimento de dieta adequada e rotina de atividade física insuficiente, como tem revelado alguns estudos ${ }^{(14,15)}$. Isto mostra a necessidade de que sejam desenvolvidos trabalhos que estabeleçam a eficácia dos referidos programas, embora a nossa realidade seja diferente dos contextos nos quais foram desenvolvidos os trabalhos analisados. Dessa forma, é possível elaborar estratégias de educação em saúde eficazes, isto é, que levem a clientela a mudanças nos hábitos cotidianos.

O desenvolvimento desse trabalho, que abordou a identificação de melhores estratégias para educação em saúde com hipertensos, apresentou algumas dificuldades como a clareza dos métodos e intervenções utilizadas, e a busca por trabalhos que apresentassem enfermeiros abordando esta temática. Porém, é indiscutível sua importância, pois foi mais um aprendizado para o desenvolvimento de pesquisas e como desenvolver estratégias para trabalhar educação em saúde com indivíduos portadores de doenças crônicas como a hipertensão arterial.

\section{REFERÊNCIAS}

1 Brasil. Ministério da Saúde. Plano de Reorganização da Atenção à Hipertensão Arterial e ao Diabetes Mellitus. Brasília (DF): Ministério da Saúde: 2001. Disponível em: URL: http://dtr2001.saude.gov.br/ bvs/publicacoes/reorganizacao_plano.pdf

2 Ribeiro AB. Atualização em hipertensão arterial: clínica, diagnóstico e terapêutica. São Paulo (SP): Atheneu, 1996.

3. Achutti AC, Achutti VAR. Aspectos epidemiológicos. In: Amodeu C Lima EG, Varquez EC. Hipertensão arterial. São Paulo (SP): Sarvier; 1997. p.11-22

4. Pierin AMG, Mion Jr D. Atuação da equipe de enfermagem na hipertensão arterial. In: Pierin AMG. Manual de EnfermagemPrograma Saúde da Família-Saúde do adulto e do idoso. Porto Alegre (RS): Sagra-Luzzato; 2001.

5. Sociedade Brasileira de Hipertensão Arterial. Diretrizes Brasileira de Hipertensão Arterial. In: Anais do Congresso da Sociedade Brasileira de Hipertensão. Campos do Jordão (SP); Fev 2002. São Paulo (SP): SBH; 2002. p. 40.
6. Zernike WBN, Henderson ABS. Evaluating tehe effectiveness of two teching strategies for patients diagnosed with hypertension. J Clin Nurs 1998; 7: 37-44

7. Araujo TL Hipertensão arterial - um problema de saúde coletiva e individual. In: Damasceno MMC, Araujo TL, Fernandes AFC. Transtornos vitais no fim do século XX: diabetes mellitus, distúrbios cardiovasculares, câncer, AIDS, tuberculose e hanseníase. Fortaleza (CE): Fundação Cearense de Pesquisa e Cultura; 1999. p. 33-8.

8. Galvão CM, Sawada NO, Mendes IAC. A busca das melhores evidências. Rev Esc Enferm USP 2003; 37(4): 43-50.

9. Polit DF, Hungler BP. Delineamento de pesquisa. In: Polit DF, Hungler BP. Fundamentos da pesquisa em enfermagem. $3^{\mathrm{a}}$ ed. Porto Alegre (RS): Artes Médicas; 1995. p.108-40.

10. Yeh GY, Einsenberg DM, Kaptchuk TJ, Phillips RS. Revisão sistemática de ervas e suplementos dietéticos para controle glicêmico em diabetes. Diabetes Care 2003; 2(3): 100-18. 
Eficácia de programas de educação em saúde para portadores de hipertensão arterial

11. Lobiondo-Wood G, Haber J. Métodos, avaliação crítica e utilização. $4^{a}$ ed. Rio de Janeiro (RJ): Guanabara Koogan; 2001.

12. Saounatsou M, Patsi O, Fasoi G, Stylianou M, Kavga A, Economou $O$, Mandi $P$, Nicolaou M. The influence of the hypertensive patient's education in compliance with their medication. Public Health Nurs 2001; 18(6): 436-42.

13. Tavares DMS, Moreira MIGB, Peres DA, Ribeiro FCSA, Dib LA. O trabalho do médico e do enfermeiro no Programa Saúde da Família.
Enfermagem Atual 2003; 18: 17-21.

14. Moreira TMM, Araújo TL de. Utilização da teoria de King na facilitação da adesão ao tratamento da hipertensão. Cogitare Enfermagem 1999; 4(1): $21-8$

15. Moreira TMM, Araújo TL, Pagliuca LMF. Alcance da teoria de King junto a famílias de pessoas portadoras de hipertensão arterial sistêmica. Rev Gaúcha Enferm 2002; 22(1): 74-89. 